\title{
Screening of compound libraries for inhibitors of Toxoplasma growth and invasion
}

\author{
Yongmei Han ${ }^{1,2}$. Oluyomi Stephen Adeyemi ${ }^{1,3} \cdot$ Mohammad Hazzaz Bin Kabir ${ }^{1} \cdot$ Kentaro Kato $^{1,4}$
}

Received: 16 December 2018 / Accepted: 20 March 2020 / Published online: 1 April 2020

(C) Springer-Verlag GmbH Germany, part of Springer Nature 2020

\begin{abstract}
Toxoplasma gondii can infect virtually all warm-blooded animals, including humans. It can differentiate between rapidly replicating tachyzoites that cause acute infection and slowly growing bradyzoites in tissue cysts. Treatment options for toxoplasmosis are challenging because current therapies cannot eradicate the latent $T$. gondii infection that is mainly caused by the bradyzoite forms. Accordingly, recurrence of infection is a problem for immunocompromised patients and congenitally infected patients. Protein kinases have been widely studied in eukaryotic cells, and while little is known about signaling in Toxoplasma infection, it is likely that protein kinases play a key role in parasite proliferation, differentiation, and probably invasion. To identify optimized new kinase inhibitors for drug development against $T$. gondii, we screened a library of kinase inhibitor compounds for anti-Toxoplasma activity and host cell cytotoxicity. Pyrimethamine served as a positive control and $0.5 \%$ DMSO was used as a negative control. Among the 80 compounds screened, 6 compounds demonstrated $\geq 80 \%$ parasite growth inhibition at concentrations at which 5 compounds did not suppress host cell viability, while 3 kinase inhibitors (Bay 11-7082, Tyrphostin AG 1295 and PD-98059) had suppressive effects individually on parasite growth and host cell invasion, but did not strongly induce bradyzoite formation.
\end{abstract}

Keywords Anti-Toxoplasma gondii $\cdot$ Bradyzoite $\cdot$ Kinase inhibitor $\cdot$ Toxoplasmosis

\section{Introduction}

Toxoplasmosis is a zoonotic infection, and cats play important roles in its spread. In humans, infection occurs upon consump-

Section Editor: Berit Bangoura

Electronic supplementary material The online version of this article (https://doi.org/10.1007/s00436-020-06673-9) contains supplementary material, which is available to authorized users.

Kentaro Kato

kentaro.kato.c7@tohoku.ac.jp

1 National Research Center for Protozoan Diseases, Obihiro University of Agriculture and Veterinary Medicine, Inada-cho, Obihiro, Hokkaido 080-8555, Japan

2 College of Animal Science and Technology, Inner Mongolia University For Nationalities, Tongliao 028000, Inner Mongolia, People's Republic of China

3 Department of Biochemistry, Medicinal Biochemistry and Toxicology Laboratory, Landmark University, PMB 1001, Ipetu Road, Omu-Aran 251101, Nigeria

4 Laboratory of Sustainable Animal Environment, Graduate School of Agricultural Science, Tohoku University, 232-3 Yomogida, Naruko-onsen, Osaki, Miyagi 989-6711, Japan tion of insufficiently cooked meat derived from infected animals or foods contaminated with soil containing Toxoplasma gondii oocysts shed from infected cats (Black and Boothroyd 2000). Primary infection of pregnant women causes miscarriage, fetal encephalopathy, convulsions, hydrocephalus, intracranial calcification, and other symptoms (Demar et al. 2012; Campos et al. 2014; Fallahi et al. 2018). Toxoplasma employs various survival strategies, such as intracellular parasitism and immune disturbance, to escape from the host's immune response, so vaccine development is extremely difficult. The current anti-protozoan drugs however do not efficiently target the bradyzoite stage, but rather may induce latent infection.

Only a limited number of drugs are available to treat toxoplasmosis patients. The antifolate medicines sulfadiazine and pyrimethamine inhibit Toxoplasma folate synthesis (Dittmar et al. 2016); however, these drugs do not kill the bradyzoite forms. Therefore, new drugs are needed.

Non-biased screening of large libraries of compounds is a common approach to identify lead compounds that can be further refined to develop novel therapeutics. Although lack of information regarding compound toxicity to the host, mechanism of action and pharmacokinetics is surmountable, 
obtaining such information can be time consuming and costly.

Protein kinases have been extensively studied in eukaryotic cells, but little is known about signal transduction in Toxoplasma infection. Therefore, the protozoan protein kinases may play important roles in protozoan infection, host cell proliferation, differentiation, and other infection steps (Peixoto et al. 2010).

Protein kinase inhibitors have been extensively studied as cancer therapeutic agents (Kannaiyan and Mahadevan 2018); some of these inhibitors have relatively few side effects, while many have made it to market. For example, piceatannol has been reported to have an inhibitory effect on malaria (Mishra et al. 1999); however, it is not known whether piceatannol can suppress Toxoplasma activity. In this study, a validated kinase inhibitor library was screened for anti-Toxoplasma activity and host cell cytotoxicity. Compounds with good antiToxoplasma activity and low host cell toxicity were then further evaluated for their effects on invasion and bradyzoite differentiation. In light of the above, we provide evidence that kinase inhibitors have anti-Toxoplasma activity in vitro.

\section{Materials and methods}

\section{Compounds}

For the preliminary screening, we used a library of 80 compounds (ENZO; CB-BML-2832J0100, $10 \mathrm{mM}$ in a 96-well plate), provided by the Cancer Research Institute of Kanazawa University, Japan. Pyrimethamine (Wako, Osaka, Japan), Tyrphostin AG 1295 (Cayman Chemical Company, USA), piceatannol (Wako, Osaka, Japan), PD-98059 (Cayman Chemical Company, USA), BAY 11-7082 (Wako, Osaka, Japan); daidzein (Wako, Osaka, Japan), apigenin (Wako, Osaka, Japan) were used for secondary screening.

\section{Host cells}

Vero cells from African green monkey kidney (Vero, RIKEN BioResource Center: RCB0001) or human foreskin fibroblast (HFF, ATCC: SCRC-1041) cells were used for parasite culture. Vero cells were cultured in Dulbecco's Modified Eagle's Medium (DMEM, Nissui Pharmaceutical, Tokyo, Japan) supplemented with 5\% FBS, 2 mM L-glutamine, and $100 \mathrm{U} / \mathrm{mL}$ penicillin-streptomycin. HFF cells were cultured in DMEM supplemented with 10\% FBS, 2 mM L-glutamine, and $100 \mathrm{U} / \mathrm{mL}$ penicillin-streptomycin.

\section{Parasite}

T. gondii $\mathrm{RH}$ strain $2 \mathrm{~F}$ expressing beta-galactosidase was used for the growth inhibition assay, and PLK/DLUC_1C9 (Sugi et al. 2014) was used for the bradyzoite differentiation assay. Host cells infected with $T$. gondii tachyzoites were passed through a 27-gauge needle to lyse them. The cell lysates were then filtered through a 5- $\mu \mathrm{m}$ filter to obtain a tachyzoite suspension free of host cell debris. The suspension was washed with fresh culture medium. Then, the parasite density was measured with a hemocytometer and adjusted for in vitro experimental infection analysis.

\section{Toxoplasma gondii growth inhibition assay}

In the preliminary screening, all compounds were screened at a concentration of $25 \mu \mathrm{M}$ for either anti-parasitic activity or host cytotoxicity to identify hit compounds (compounds with anti-parasitic action $\geq 80 \%$ but no host cytotoxicity). After the preliminary screening, hit compounds were further screened for dose-response effects at various concentrations between 0 and $25 \mu \mathrm{M}$.

To determine the anti- $T$. gondii activities of the test compounds, in vitro growth inhibition assays were performed as previously described (Ishiwa et al. 2013; Adeyemi et al. 2017). Briefly, freshly purified parasite suspension and the test compounds (freshly reconstituted in culture medium prior to use) were simultaneously added and incubated in growing HFF monolayers. The multiplicity of infection (MOI) was 1:5 (parasite:host cell ratio). Medium-treated cells served as a negative control, while the "media only" well was used to correct for any background signal. Pyrimethamine was included as a reference compound to serve as a positive control. After a 48 -h incubation at $37{ }^{\circ} \mathrm{C}$ in a $5 \% \mathrm{CO}_{2}$ atmosphere, the viability of the $\mathrm{RH}-2 \mathrm{~F}$ parasite strain was determined by assaying for galactosidase activity by using a Beta-Glo luminescent assay kit (Promega, Madison, USA). The assay was performed in triplicate and repeated three times. All experiments were performed in 96-well optical bottom plates (Nunc; Fisher Scientific, Pittsburgh, USA) unless otherwise stated.

\section{Cytotoxicity assay}

Vero cells were maintained in DMEM (Nissui, Tokyo, Japan) supplemented with $2 \mathrm{mM}$ L-glutamine, $5 \%$ (v/v) FCS (Gibco, Invitrogen, $\mathrm{UK}$ ), and $100 \mathrm{U} / \mathrm{mL}$ penicillin-streptomycin (Biowhittaker, UK). Cells were grown to confluence at $37{ }^{\circ} \mathrm{C}$ in a $5 \% \mathrm{CO}_{2}$ atmosphere. Confluent cells were subcultured and seeded in 96-well plates (Nunc; Fisher Scientific, Pittsburgh, USA) at a density of $1 \times 10^{5}$ cells per well. After a $24-\mathrm{h}$ incubation at $37{ }^{\circ} \mathrm{C}$ in a $5 \% \mathrm{CO}_{2}$ atmosphere, test compounds at various concentrations $(0.78-$ $100 \mu \mathrm{M}$ ) were added. The treated cells were incubated for 72-h under normal tissue culture conditions and cell viabilities were measured by using a Cell-Titer Glo kit (Promega) following the manufacturer's instructions. Control wells were 
treated with DMSO alone in culture medium to calculate the $100 \%$ viability for each cell type.

\section{Toxoplasma gondii red green invasion inhibition assay}

Treatment of tachyzoites and invasion experiments were performed as described previously with slight modification (Robert-Gangneux et al. 2000). In this experiment, tachyzoites were treated with $25 \mu \mathrm{M}$ of the compounds (single concentration). For immunofluorescence assay, extracellular parasites were stained by anti-SAG1 monoclonal mouse antibody (TP3; HyTest), while Alexa-546 conjugated goat anti-mouse $\mathrm{IgG}$ was used as secondary antibody. After washing and permeabilization, intracellular parasites were stained by antiGAP45 polyclonal rabbit antibody (a gift from Dr. Dominique Soldati-Favre; University of Geneva, Switzerland) and Alexa 488-conjugated goat anti-rabbit IgG was used as secondary antibody, while DAPI was used for nuclear staining.

\section{Bradyzoite differentiation assay}

To measure the effect of the kinase inhibitors on bradyzoite induction, we followed the protocol described by Murata et al. (2017). Briefly, purified PLK/DLUC_1C9 parasites (Sugi et al. 2014) were inoculated at a concentration of 20,000 parasites/well into 12-well plates containing confluent monolayers of HFF cells. After a $24-\mathrm{h}$ incubation at $37^{\circ} \mathrm{C}, 5 \% \mathrm{CO}_{2}$, the infection medium was removed and replaced with fresh medium containing the hit compounds $(25 \mu \mathrm{M})$, and the cells were incubated for $48 \mathrm{~h}$ at $37{ }^{\circ} \mathrm{C}, 5 \% \mathrm{CO}_{2}$. Thereafter, the medium in each well was removed, and the contents were lysed using $200 \mu \mathrm{L}$ of Passive Lysis Buffer (Promega). The lysates was stored at $-80^{\circ} \mathrm{C}$. For the luciferase assay, $25 \mu \mathrm{L}$ of lysate in a 96-well plate (white) was mixed with an equal volume of Dual Glo Luciferase Assay Reagent and Dual Glo Stop and Glo Reagent to measure the firefly luciferase and Renilla activities in accordance with the manufacturer's instructions (Promega). PLK/DLUC_1C9 parasite expresses firefly luciferase under the control of the bradyzoite-specific BAG-1 promoter (bradyzoite) and Renilla luciferase under the control of the $\alpha$-tubulin promoter (total parasite). The ratio of bradyzoite-inducing activity relative to total parasites was calculated. Pyrimethamine $(10 \mu \mathrm{M})$ was included as a positive control (100\% activity).

\section{Statistical analyses}

The Dunnett's post hoc test (GraphPad Prism software) was used to compare between more than two groups. $p$ values $\leq$ 0.05 were considered to be statistically significant. The $\mathrm{IC}_{50}$ and $\mathrm{EC}_{50}$ values were determined by analysis of dose- response inhibition by using GraphPad prism. The ratio of the $\mathrm{IC}_{50}$ for mammalian cellular toxicity to that of $\mathrm{EC}_{50}$ for anti-parasite activity was taken as the selective index (SI) for the various compounds.

\section{Results}

\section{Screening of the kinase inhibitor library for anti-Toxoplasma effects}

A library of 80 compounds was preliminarily screened at a concentration of $25 \mu \mathrm{M}$ for their $T$. gondii growth inhibitory effects as well as for host cytotoxicity. Of the eighty (80) compounds, six (6) compounds showed $>80 \%$ Toxoplasma gondii growth inhibition activity, and only five (5) of these displayed low host cell toxicity relative to the positive control (Fig. 1a and Fig. 2). Tyrphostin AG1295 exhibits considerable host cell cytotoxicity. In this experiment, the galactosidase activity of the negative control wells (only DMSO) was calculated as $0 \%$ inhibition and that of the Vero cell only wells (no parasite) was calculated as $100 \%$ inhibition (no galactosidase activity). This result suggests that these compounds could be considered leads for anti-Toxoplasma therapy. More so, compounds showed low host cell toxicity (Fig. 2).

To clarify the dose-response efficacy of these compounds, we added them to parasite cultures after host cell invasion. When the compounds were added after the 2-h invasion window, the same 6 compounds (Supplementary Fig. 1) inhibited parasite growth in a dose-dependent manner (Fig. 1b). The parasite growth rate and the host cell viability are presented in Table 1. Meanwhile, the $50 \%$ effective concentration $\left(\mathrm{EC}_{50}\right)$ of the 6 compounds ranged from 1.5-11 $\mu \mathrm{M}$ (Table 2). Next, we calculated the $50 \%$ inhibitory concentration $\left(\mathrm{IC}_{50}\right)$ value against Vero cells, and thereby estimated selectivity index for each compound as a measure of therapeutic efficacy (Table 2). Together, the data suggest that five (5) of the compounds exhibited low host cell toxicity, thus indicating anti-Toxoplasma specificity. Only Tyrphostin AG1295 shows host cellular toxicity. Additionally, the low cytotoxic action in host cells by these compounds suggests exclusion of general cellular toxicity.

\section{Kinase inhibitors inhibit parasite invasion}

To evaluate whether the 6 compounds inhibit parasite invasion, we performed an assay to determine inhibition of invasion. Compounds (final concentration $25 \mu \mathrm{M}$ ) were diluted with RH-2F strain in medium and incubated $30 \mathrm{~min}$ at $37{ }^{\circ} \mathrm{C}$ and $5 \% \mathrm{CO}_{2}$ before the addition of confluent Vero cells. After the 2-h invasion period, the extracellular parasites and compounds were washed away and replaced with new medium, and the incubation was continued for a further 12-h. Of the 6 


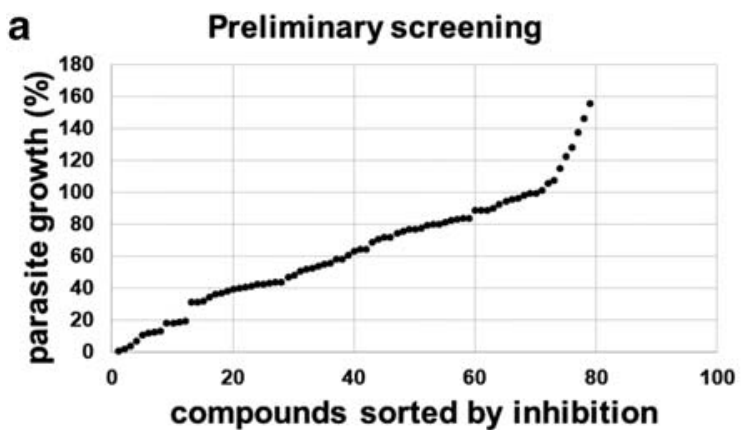

b
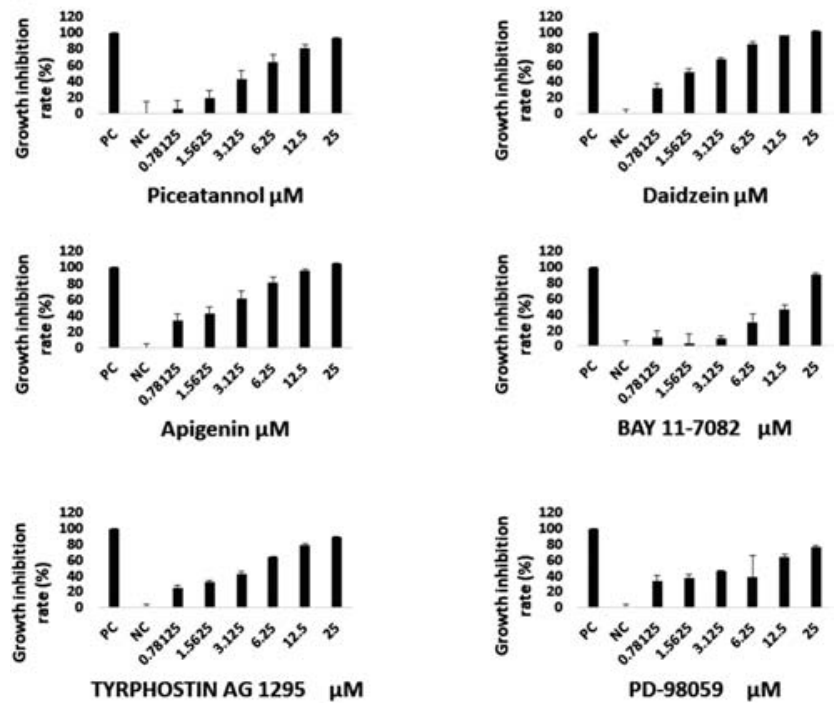

Fig. 1 Screening for anti-Toxoplasma activity (a) T. gondii $\mathrm{RH}$ strain $2 \mathrm{~F}$ infected Vero cells were incubated with kinase inhibitors at $25 \mu \mathrm{M}$ test compounds ( 80 compounds) for $48 \mathrm{~h}$. The galactosidase activity expressed by $T$. gondii $\mathrm{RH}-2 \mathrm{~F}$ parasite was measured, and the inhibition rate was calculated with DMSO as a negative control ( $0 \%$ inhibition). (b) Dose response curves of six kinase inhibitors. Vero cells infected with T. gondii $\mathrm{RH}$ strain $2 \mathrm{~F}$ parasites were incubated with various concentrations of the test compounds, and parasite growth was measured by using the $\beta$-galactosidase assay. Inhibition rates were calculated by using DMSO as a negative control (0\% inhibition) and only Vero cells as a positive control (100\% inhibition)
Table 1 Hit compounds from the primary screening

\begin{tabular}{lll}
\hline Compounds & $\begin{array}{l}\text { Parasite growth rate } \\
(\%)^{\mathrm{a}}\end{array}$ & $\begin{array}{l}\text { Host cell viability } \\
(\%)^{\mathrm{b}}\end{array}$ \\
\hline Piceatannol & 14.1 & 90.37 \\
Daidzein & 4.2 & 102.3 \\
Apigenin & 2.4 & 101.6 \\
BAY11-7082 & 10.5 & 107.4 \\
Tyrphostin AG1295 & 9.0 & 51.5 \\
PD98059 & 10.9 & 88.1
\end{tabular}

${ }^{\text {a }}$ Parasite growth rates from the preliminary screening of $T$. gondii $\mathrm{RH}$ strain 2F-infected Vero cells with test compounds at a concentration of $25 \mu \mathrm{M}$. The DMSO control was set as $100 \%$

${ }^{\mathrm{b}}$ Host cell viability values from the preliminary screening of Vero cells with compounds at a concentration of $25 \mu \mathrm{M}$. The wells with only Vero cells was set as $100 \%$ cell viability

kinase inhibitors, 4 compounds inhibited $T$. gondii invasion compared with the negative control of $0.5 \%$ DMSO (Table 3 , Fig. 3).

\section{Effects of the kinase inhibitors on $T$. gondii bradyzoites}

To confirm whether these kinase inhibitors have bradyzoiteinducing activity, we assayed for BAG-1 promoter activity, which is a reflection of the ratio of bradyzoites to total parasites. We used the dual luciferase reporting parasite PLKDLUC_1C9 (Sugi et al. 2014), which expresses firefly luciferase activity under the control of the BAG-1 promoter and Renilla luciferase under the control of the constitutively active $\alpha$-tubulin promoter. We tested the 6 compounds and found that 4 of the compounds caused $\leq 30 \%$ increase in BAG-1 promoter (Fig. 4), relative to $10 \mu \mathrm{M}$ pyrimethamine (taken as $100 \%$ ). These findings suggest that these 4 compounds might have low bradyzoite-inducing effects. (Fig. 4). We also tested the drugs for bradyzoite inhibition assay (after 3 days of
Fig. 2 Host cell viability. Six (6) compounds were then screened for effects on host cell viability (see "Materials and Methods" section). Compounds were screened at a concentration of $0.78-100 \mu \mathrm{M}$. Only the wells of Vero cells were calculated as $100 \%$ cell viability

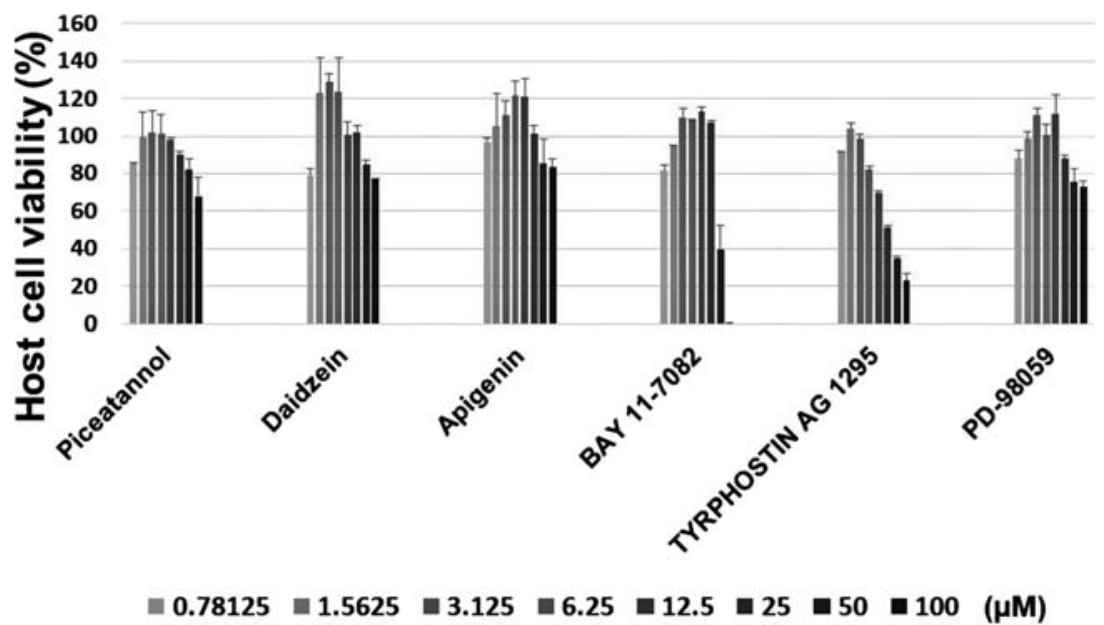


Table $2 \mathrm{EC}_{50}$ and $\mathrm{IC}_{50}$ values and selectivity indices for the six (6) kinase inhibitors

\begin{tabular}{llll}
\hline Compounds & $\begin{array}{l}\mathrm{EC}_{50} \text { (anti-parasite) } \\
(\mu \mathrm{M})\end{array}$ & $\begin{array}{l}\mathrm{IC}_{50} \text { (host cell) } \\
(\mu \mathrm{M})\end{array}$ & $\begin{array}{l}\text { Selectivity index } \\
\left(\mathrm{IC}_{50} / \mathrm{EC}_{50}\right)\end{array}$ \\
\hline Piceatannol & 4.2 & $>100$ & $>23.8$ \\
Daidzein & 1.5 & $>100$ & $>66.6$ \\
Apigenin & 2.0 & $>100$ & $>50$ \\
BAY11-7082 & 10.9 & 51.8 & 4.8 \\
Tyrphostin AG 1295 & 5.0 & 28.5 & 5.7 \\
PD98059 & 7.3 & $>100$ & $>13.7$ \\
\hline
\end{tabular}

$\mathrm{EC}_{50}, 50 \%$ effective concentration

$\mathrm{IC}_{50}, 50 \%$ inhibitory concentration inducing bradyzoites, followed by treatment with $25 \mu \mathrm{M}$ of the drugs for additional 3 days), but found the drugs showed no bradyzoite-killing activity compared with $0.5 \%$ DMSO (data not shown).

\section{Discussion}

In this study, we screened a library of kinase inhibitor compounds for their ability to inhibit Toxoplasma growth. We found 6 kinase inhibitors with $>80 \%$ Toxoplasma gondii growth inhibitory activity, and five (5) of these compounds had low host cell cytotoxicity relative to the inhibitory dose against the parasite; therefore, they could be considered as leads for anti-Toxoplasma therapy. Tyrphostin AG1295 exhibits considerable host cell cytotoxicity. Of the 6 kinase inhibitors, 4 compounds (piceatannol, Bay 11-7082, Tyrphostin AG 1295, and PD-98059) showed Toxoplasma gondii invasion inhibition activity. The kinase inhibitors daidzein, Bay 11-7082, Tyrphostin AG 1295, and PD-98059 strongly inhibited Toxoplasma growth, but lacked strong bradyzoiteinducing capacity at the tested concentration of $25 \mu \mathrm{M}$. Although we have not yet assessed the mechanism by which these anti-Toxoplasma compounds block parasite growth, the mechanism may reveal a new target for the control of T. gondii.

While therapy exists for the treatment of toxoplasmosis, it is suboptimal and there is a need for new therapeutic agents with fewer side effects and for agents that are active against

Table 3 Parasite invasion inhibition rates for the hit compounds

\begin{tabular}{ll}
\hline Compound & Invasion inhibition rate (\%) \\
\hline Piceatannol & 50.4 \\
Daidzein & 8.5 \\
Apigenin & 14.1 \\
BAY 11-7082 & 79.6 \\
Tyrphostin AG1295 & 62.8 \\
PD-98059 & 51.7 \\
\hline
\end{tabular}

latent infection. The use of a bradyzoite reporter parasite facilitates the evaluation of compounds for their effects against both tachyzoites and bradyzoites. We identified four compounds (daidzein, Bay 11-7082, Tyrphostin AG 1295, and PD-98059) that showed promising anti-Toxoplasma activity but did not induce bradyzoite formation. This is desirable, given that current treatment options, like pyrimethamine, do not prevent bradyzoite formation, and more importantly, there is currently no treatment option to combat bradyzoites formed in tissue cysts. The screening method described in this study represents a strategy to identify anti-Toxoplasma compounds that have both Toxoplasma growth inhibition and bradyzoite induction capability. Several drugs that target these latent parasites have recently been identified and include a bumped kinase inhibitor (Doggett et al. 2014) and protein kinase inhibitor (Wei and Liu 2013) that targets T. gondii CDPK1 (Sugi et al. 2010), and endochin-like quinolones (Doggett et al. 2012), which inhibit the cytochrome bc1 complex. The activity of these compounds toward bradyzoites suggests that therapeutic agents can be found to treat latent infection caused by this parasite. Additional screens using the approach presented here should help identify more lead compounds, and it is likely that one of these leads will result in a clinically effective new therapy for the treatment of the chronic infection. Furthermore, to meet the need for new antimicrobial therapies, we are driving the screening of wider ranges of chemical compounds to identify those with anti-parasitic activity (Adeyemi et al. 2017, 2018).

Four kinase inhibitors demonstrated parasite invasion inhibition effects at a concentration of $25 \mu \mathrm{M}$, suggesting antiparasite-specific action. Therefore, these compounds may be viable leads for $T$. gondii therapy. By contrast, pyrimethamine, which is currently used to treat toxoplasmosis, showed no activity against parasite invasion. Most of the hit compounds identified in this study have not been previously reported to have anti-T. gondii activity, except for daidzein which were reported previously by us (Adeyemi et al. 2018). Further study is required to unravel the mechanistic basis for the antiT. gondii effects of these compounds. In the conclusion, of the 80 kinase inhibitors screened for Toxoplasma growth 
Fig. 3 Invasion inhibitory effects of the 6 kinase inhibitors. Shown are invasion inhibition rates for $0.5 \%$ DMSO (control) and kinase inhibitors at $25 \mu \mathrm{M}$. Kinase inhibitors in medium were used to treat the parasite ( $T$. gondii $\mathrm{RH}$ strain $2 \mathrm{~F}$ ) for $30 \mathrm{~min}$ at $37^{\circ} \mathrm{C} 5 \%$ $\mathrm{CO}_{2}$ and parasites were then added to the wells for $2 \mathrm{~h}$ in the presence of kinase inhibitors.

Thereafter, extracellular parasites were washed away and new medium was added. Infected host cells were incubated for $12 \mathrm{~h}$. The cells were fixed in $4 \%$ paraformaldehyde for $30 \mathrm{~min}$ then washed 3 times and kept in PBS followed by IFA staining. The DMSO control was set as $100 \%$. Mean \pm SD from triplicate experiments are shown. The statistical difference between the DMSO control and each compound was evaluated by using Dunnett's test. $* p<0.05$, n.s, not significant
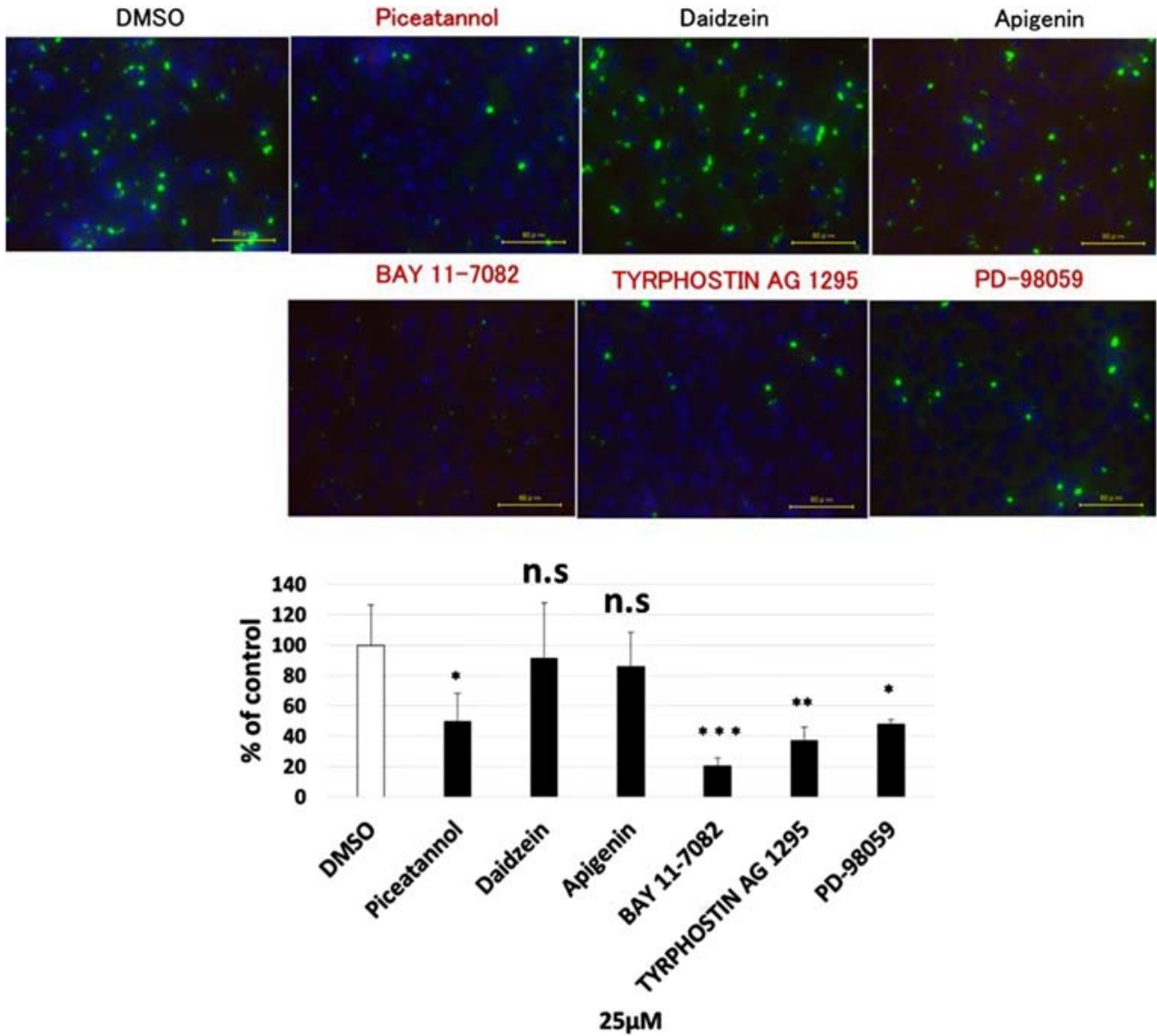

inhibition, 6 compounds showed $\geq 80 \%$ inhibitory potential against the parasite growth, and 5 of these compounds demonstrated low host cell cytotoxicity. Among them, 4 kinase inhibitors inhibit parasite invasion process in vitro. Four kinase inhibitors have also low bradyzoite-inducing activity, while 3 kinase inhibitors (Bay 11-7082, Tyrphostin AG

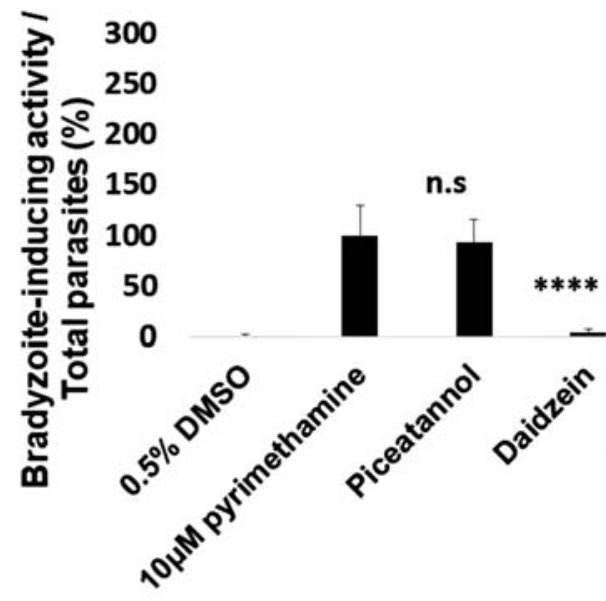

n.s

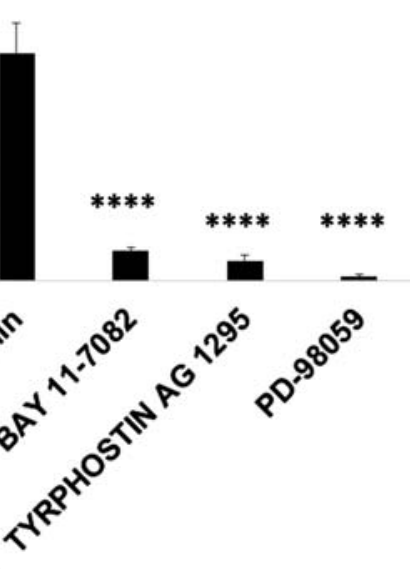

$25 \mu \mathrm{M}$

1 promoter, by the Renilla luciferase activity driven by the $\alpha$-tubulin promoter. Relative BAG-1 promoter activity was compared with that of pyrimethamine-treated samples as shown. Mean \pm SD are from triplicate experiments. The statistical difference between the DMSO solvent control group (tachyzoite) and each compound was evaluated by using Dunnett's test, $* * * * p<0.0001$, n.s: not significant 
1295, and PD-98059) had suppressive effects individually on parasite growth, invasion inhibition, and bradyzoite-inducing activity.

Acknowledgments The kinase inhibitor library was provided by the Cancer Research Institute of Kanazawa University.

Funding information This study was supported by grants-in-aid for Young Scientists, Exploratory Research, and Scientific Research on Innovative Areas (3407); Scientific Research (B); and (C) from the Ministry of Education, Culture, Science, Sports, and Technology (MEXT) of Japan; by the Program to Disseminate Tenure Tracking System from the Japan Science and Technology Agency (JST); and by the Livestock Promotional Subsidy from the Japan Racing Association.

\section{Compliance with ethical standards}

Conflict of interest The authors declare that they have no conflict of interest.

\section{References}

Adeyemi OS, Molina MT, Eseola AO, Fonseca-Berzal C, Gomez-Barrio A (2017) New imidazole-based compounds active against Trypanosoma cruzi. Comb Chem High Throughput Screen 20(1): 20-24. https://doi.org/10.2174/1386207320666170110141907

Adeyemi OS, Sugi T, Han Y, Kato K (2018) Screening of chemical compound libraries identified new anti-Toxoplasma gondii agents. Parasitol Res 117(2):355-363. https://doi.org/10.1007/s00436-017$5698-1$

Black MW, Boothroyd JC (2000) Lytic cycle of toxoplasma gondii. Microbiol Mol Biol Rev 64(3):607-623

Campos FA, Andrade GM, Lanna Ade P, Lage BF, Assumpcao MV, Pinto JA (2014) Incidence of congenital toxoplasmosis among infants born to HIV-coinfected mothers: case series and literature review. Braz J Infect Dis 18(6):609-617. https://doi.org/10.1016/j. bjid.2014.05.008

Demar M, Demar M, Hommel D, Djossou F, Peneau C, Boukhari R, Louvel D, Bourbigot AM, Nasser V, Ajzenberg D, Darde ML, Carme B (2012) Acute toxoplasmoses in immunocompetent patients hospitalized in an intensive care unit in French Guiana. Clin Microbiol Infect 18(7):E221-E231. https://doi.org/10.1111/j.14690691.2011.03648.x

Dittmar AJ, Drozda AA, Blader IJ (2016) Drug repurposing screening identifies novel compounds that effectively inhibit Toxoplasma gondii growth. mSphere 1(2). https://doi.org/10.1128/mSphere. 00042-15

Doggett JS, Nilsen A, Forquer I, Wegmann KW, Jones-Brando L, Yolken RH, Bordon C, Charman SA, Katneni K, Schultz T, Burrows JN,
Hinrichs DJ, Meunier B, Carruthers VB, Riscoe MK (2012) Endochin-like quinolones are highly efficacious against acute and latent experimental toxoplasmosis. Proc Natl Acad Sci U S A 109(39):15936-15941. https://doi.org/10.1073/pnas.1208069109

Doggett JS, Ojo KK, Fan E, Maly DJ, Van Voorhis WC (2014) Bumped kinase inhibitor 1294 treats established Toxoplasma gondii infection. Antimicrob Agents Chemother 58(6):3547-3549. https://doi. org/10.1128/AAC.01823-13

Fallahi S, Rostami A, Nourollahpour Shiadeh M, Behniafar H, Paktinat S (2018) An updated literature review on maternal-fetal and reproductive disorders of Toxoplasma gondii infection. J Gynecol Obstet Hum Reprod 47(3):133-140. https://doi.org/10.1016/j.jogoh.2017. 12.003

Ishiwa A, Kobayashi K, Takemae H, Sugi T, Gong H, Recuenco FC, Murakoshi F, Inomata A, Horimoto T, Kato K (2013) Effects of dextran sulfates on the acute infection and growth stages of Toxoplasma gondii. Parasitol Res 112(12):4169-4176. https://doi. org/10.1007/s00436-013-3608-8

Kannaiyan R, Mahadevan D (2018) A comprehensive review of protein kinase inhibitors for cancer therapy. Expert Rev Anticancer Ther 18(12):1249-1270. https://doi.org/10.1080/14737140.2018. 1527688

Mishra NC, Sharma M, Sharma A (1999) Inhibitory effect of piceatannol, a protein tyrosine kinase inhibitor, on asexual maturation of Plasmodium falciparum. Indian J Exp Biol 37(4):418-420

Murata Y, Sugi T, Weiss LM, Kato K (2017) Identification of compounds that suppress Toxoplasma gondii tachyzoites and bradyzoites. PLoS One 12(6):e0178203. https://doi.org/10.1371/journal.pone.0178203

Peixoto L, Chen F, Harb OS, Davis PH, Beiting DP, Brownback CS, Ouloguem D, Roos DS (2010) Integrative genomic approaches highlight a family of parasite-specific kinases that regulate host responses. Cell Host Microbe 8(2):208-218

Robert-Gangneux F, Creuzet C, Dupouy-Camet J, Roisin M-P (2000) Involvement of the mitogen-activated protein (MAP) kinase signalling pathway in host cell invasion by Toxoplasma gondii. Parasite 7: 95-101

Sugi T, Kato K, Kobayashi K, Watanabe S, Kurokawa H, Gong H, Pandey K, Takemae H, Akashi H (2010) Use of the kinase inhibitor analog 1NM-PP1 reveals a role for Toxoplasma gondii CDPK1 in the invasion step. Eukaryot Cell 9(4):667-670

Sugi T, Masatani T, Murakoshi F, Kawazu S, Kato K (2014) Microplate assay for screening Toxoplasma gondii bradyzoite differentiation with DUAL luciferase assay. Anal Biochem 464:9-11. https://doi. org/10.1016/j.ab.2014.06.018

Wei F, Liu Q (2013) Protein kinase of Toxoplasma gondii: functions and drug targets. Parasitol Res 112:2121-2129. https://doi.org/10.1007/ s00436-013-3451-y

Publisher's note Springer Nature remains neutral with regard to jurisdictional claims in published maps and institutional affiliations. 